TRANSACTIONS OF THE

AMERICAN MATHEMATICAL SOCIETY

Volume 359, Number 9, September 2007, Pages 4449-4465

S 0002-9947(07)04186-4

Article electronically published on March 20, 2007

\title{
FREE INTERPOLATION BY NONVANISHING ANALYTIC FUNCTIONS
}

\author{
KONSTANTIN DYAKONOV AND ARTUR NICOLAU
}

\begin{abstract}
We are concerned with interpolation problems in $H^{\infty}$ where the values prescribed and the function to be found are both zero-free. More precisely, given a sequence $\left\{z_{j}\right\}$ in the unit disk, we ask whether there exists a nontrivial minorant $\left\{\varepsilon_{j}\right\}$ (i.e., a sequence of positive numbers bounded by 1 and tending to 0 ) such that every interpolation problem $f\left(z_{j}\right)=a_{j}$ has a nonvanishing solution $f \in H^{\infty}$ whenever $1 \geq\left|a_{j}\right| \geq \varepsilon_{j}$ for all $j$. The sequences $\left\{z_{j}\right\}$ with this property are completely characterized. Namely, we identify them as "thin" sequences, a class that arose earlier in Wolff's work on free interpolation in $H^{\infty} \cap \mathrm{VMO}$.
\end{abstract}

\section{INTRODUCTION AND RESULTS}

Let $H^{\infty}$ stand for the algebra of all bounded analytic functions on the disk $\mathbb{D}:=\{z \in \mathbb{C}:|z|<1\}$. A sequence $\left\{z_{j}\right\} \subset \mathbb{D}$ is called an interpolating sequence (for $H^{\infty}$ ) if each interpolation problem

$$
f\left(z_{j}\right)=a_{j} \quad(j=1,2, \ldots),
$$

with an arbitrary data sequence $\left\{a_{j}\right\} \in \ell^{\infty}$, has a solution $f \in H^{\infty}$. By a classical theorem of Carleson (see [4], Chapter VII), $\left\{z_{j}\right\}$ is an interpolating sequence if and only if

$$
\inf _{j} \prod_{k: k \neq j}\left|\frac{z_{j}-z_{k}}{1-\bar{z}_{j} z_{k}}\right|>0 .
$$

In this paper, we investigate the possibility - or its failure - to interpolate nonvanishing data sequences $\left\{a_{j}\right\} \in \ell^{\infty}$ (i.e., the ones satisfying $a_{j} \neq 0$ for all $j$ ) by nonvanishing (i.e., zero-free) functions $f \in H^{\infty}$.

Let us begin with a few observations. First of all, if the (bounded) values $\left\{a_{j}\right\}$ are "large" in the sense that

$$
\inf _{j}\left|a_{j}\right|>0
$$

Received by the editors October 11, 2004 and, in revised form, October 1, 2005.

2000 Mathematics Subject Classification. Primary 46J15, 30D50, 30H05.

Key words and phrases. Nonvanishing analytic functions, thin interpolating sequences.

Both authors were supported by the European Community's Human Potential Program under contract HPRN-CT-2000-00116 (Analysis and Operators). The first author was also supported by DGICYT Grant MTM2005-08984-C02-02, CIRIT Grant 2005-SGR-00611, Grant 02-01-00267 from the Russian Foundation for Fundamental Research, and by the Ramón y Cajal program (Spain). The second author was supported by DGICYT Grant MTM2005-00544 and CIRIT Grant 2005-SGR-00774. 
then it is certainly possible to find a nonvanishing - and even invertible - function $f \in H^{\infty}$ solving (1.1), whenever $\left\{z_{j}\right\}$ is an interpolating sequence. To this end, it suffices to put $f=e^{g}$, where $g \in H^{\infty}$ interpolates the bounded values $\log a_{j}$ at $z_{j}$ (the logarithms being suitably defined).

Actually, (1.3) is also necessary in order that for each interpolating sequence $\left\{z_{j}\right\}$ there exists a nonvanishing solution $f \in H^{\infty}$ to (1.1). To see why, note that if $f \in H^{\infty}$ has no zeros in $\mathbb{D}$, then $1 / f$ is in the Nevanlinna class

$$
\mathcal{N}:=\left\{g: g \text { analytic in } \mathbb{D}, \sup _{0<r<1} \int_{\partial \mathbb{D}} \log ^{+}|g(r \zeta)||d \zeta|<\infty\right\}
$$

(cf. 4], Chapter II, Section 5). Hence

$$
\log ^{+} \frac{1}{|f(z)|}=O\left(\frac{1}{1-|z|}\right), \quad z \in \mathbb{D}
$$

and we arrive at the necessary condition

$$
\log ^{+} \frac{1}{\left|a_{j}\right|}=O\left(\frac{1}{1-\left|z_{j}\right|}\right) .
$$

Now if $\inf _{j}\left|a_{j}\right|=0$, then we can find a subsequence $\left\{a_{j_{k}}\right\}$ with $\left|a_{j_{k}}\right|<\exp \left(-3^{k}\right)$. This done, (1.4) will fail for any interpolating sequence $\left\{z_{j}\right\}$ satisfying $1-\left|z_{j_{k}}\right|=$ $2^{-k}$.

On the other hand, if (1.1) is required to have a nonvanishing $H^{\infty}$-solution for each nonvanishing data sequence $\left\{a_{j}\right\} \in \ell^{\infty}$, then $\left\{z_{j}\right\}$ must be a finite set (so, again, we are left with the trivial case). Indeed, for any infinite sequence $\left\{z_{j}\right\} \subset \mathbb{D}$, there are nonzero $a_{j}$ 's that violate (1.4).

These observations probably answer the most naive questions that come to mind, and they give us an idea of what the right question might be. First of all, we know that only "small" data sequences (i.e., the ones with $\inf _{j}\left|a_{j}\right|=0$ ) are of interest. Besides, we have seen that the admissible decay rate for $\left\{a_{j}\right\}$ must depend on $\left\{z_{j}\right\}$. Also, of course, we would like to deal with a "free interpolation" problem, which means that the $a_{j}$ 's to be interpolated should be described in terms of their moduli only. This said, the following notion of a "nonvanishing interpolation sequence" comes out in a fairly natural way.

Definition 1.1. (a) A sequence $\left\{\varepsilon_{j}\right\} \subset \mathbb{R}$ will be called a minorant if $0<\varepsilon_{j}<1$ for all $j$ and if $\lim _{j \rightarrow \infty} \varepsilon_{j}=0$.

(b) A sequence $\left\{z_{j}\right\} \subset \mathbb{D}$ is said to be a nonvanishing interpolation sequence (an NVI-sequence, for short) if there exists a minorant $\left\{\varepsilon_{j}\right\}$ such that every interpolation problem (1.1) with

$$
1 \geq\left|a_{j}\right| \geq \varepsilon_{j} \quad(j=1,2, \ldots)
$$

has a nonvanishing solution $f \in H^{\infty}$. Furthermore, we say in this case that the minorant $\left\{\varepsilon_{j}\right\}$ is associated with $\left\{z_{j}\right\}$ (or that $\left\{\varepsilon_{j}\right\}$ is a minorant for $\left\{z_{j}\right\}$ ).

It is clear that the NVI-sequences are contained among the (usual) interpolating sequences. Indeed, given an arbitrary data sequence $\left\{b_{j}\right\}$ with $\sup _{j}\left|b_{j}\right| \leq 1$, the numbers $a_{j}:=\frac{1}{3}\left(2+b_{j}\right)$ satisfy $1 \geq\left|a_{j}\right| \geq \frac{1}{3}$; hence (1.5) holds for all but finitely many $j$ 's whenever $\left\{\varepsilon_{j}\right\}$ is a minorant for $\left\{z_{j}\right\}$. Now if $f \in H^{\infty}$ is a solution of (1.1), then $g:=3 f-2$ is in $H^{\infty}$ and interpolates $b_{j}$ at $z_{j}$.

At the same time, it turns out that NVI-sequences are, in a sense, "thinner" than generic interpolating sequences. While the exact meaning of this is given by 
Theorem 1.3 below, a preliminary result to that effect can be quickly derived from Harnack's inequality. In fact, if $f \in H^{\infty}$ is a nonvanishing solution to (1.1), then $u(z):=\log \left(\|f\|_{\infty} /|f(z)|\right)$ is a positive harmonic function on $\mathbb{D}$. Now Harnack's inequality, in its invariant form, yields

$$
\frac{1-\rho(z, w)}{1+\rho(z, w)} \leq \frac{u(z)}{u(w)} \leq \frac{1+\rho(z, w)}{1-\rho(z, w)} \quad(z, w \in \mathbb{D}),
$$

where $\rho(z, w):=|z-w| /|1-\bar{z} w|$ is the pseudohyperbolic distance between the two points. Consequently,

$$
\frac{1-\rho\left(z_{j}, z_{k}\right)}{1+\rho\left(z_{j}, z_{k}\right)} \leq \frac{\log \left(\|f\|_{\infty} /\left|a_{j}\right|\right)}{\log \left(\|f\|_{\infty} /\left|a_{k}\right|\right)} \leq \frac{1+\rho\left(z_{j}, z_{k}\right)}{1-\rho\left(z_{j}, z_{k}\right)}
$$

for all $j$ and $k$. Using (1.6), we shall now verify that

$$
\inf _{k: k \neq j} \rho\left(z_{j}, z_{k}\right) \rightarrow 1 \quad \text { as } \quad j \rightarrow \infty
$$

whenever $\left\{z_{j}\right\}$ is an NVI-sequence. Indeed, assuming the contrary, we can find an infinite set of indices $\mathcal{J} \subset \mathbb{N}$ and a constant $c<1$ so that for each $j \in \mathcal{J}$ there is a $k=k(j)$ with $\rho\left(z_{j}, z_{k}\right)<c$. Replacing $\mathcal{J}$ by a suitable subset thereof, we may further assume that $k(j) \notin \mathcal{J}$ for all $j \in \mathcal{J}$. Given a minorant $\left\{\varepsilon_{j}\right\}$ associated with $\left\{z_{j}\right\}$, define

$$
a_{j}:= \begin{cases}\varepsilon_{j} & \text { if } j \in \mathcal{J} \\ 1 & \text { otherwise }\end{cases}
$$

and let $f \in H^{\infty}$ be a nonvanishing function solving (1.1). Applying the right-hand inequality in (1.6) with $j \in \mathcal{J}$ and $k=k(j)$, we now get

$$
\frac{\log \left(\|f\|_{\infty} / \varepsilon_{j}\right)}{\log \|f\|_{\infty}} \leq \frac{1+c}{1-c}, \quad j \in \mathcal{J} .
$$

This contradicts the fact that $\varepsilon_{j} \rightarrow 0$, and (1.7) is thereby established.

It turns out that the "right" thinness condition we need is stronger than (1.7). We proceed by introducing (or rather recalling) the appropriate notion of a thin sequence.

Definition 1.2. A sequence $\left\{z_{j}\right\} \subset \mathbb{D}$ is called thin if

$$
\lim _{j \rightarrow \infty} \prod_{k: k \neq j}\left|\frac{z_{j}-z_{k}}{1-\bar{z}_{j} z_{k}}\right|=1 .
$$

We are now in a position to state our main result.

Theorem 1.3. A sequence $\left\{z_{j}\right\} \subset \mathbb{D}$ is an NVI-sequence if and only if it is thin.

We further recall the notation $\mathcal{N}$ for the Nevanlinna class, and we remark that the family $\{1 / f\}$, where $f$ ranges over the nonvanishing $H^{\infty}$-functions, coincides with the set

$$
\left\{g \in \mathcal{N}: \inf _{z \in \mathbb{D}}|g(z)|>0\right\} .
$$

Consequently, the preceding result admits an immediate restatement in terms of the Nevanlinna class. 
Proposition 1.4. Given a sequence $\left\{z_{j}\right\} \subset \mathbb{D}$, the following are equivalent:

(1) One can find a sequence $\left\{M_{j}\right\} \subset(1, \infty)$ with $\lim _{j \rightarrow \infty} M_{j}=\infty$ and a number $\delta>0$ so that every interpolation problem

$$
g\left(z_{j}\right)=b_{j} \quad \text { with } \quad 1 \leq\left|b_{j}\right| \leq M_{j} \quad(j=1,2, \ldots)
$$

has a solution $g \in \mathcal{N}$ satisfying $|g(z)| \geq \delta$ for all $z \in \mathbb{D}$.

(2) $\left\{z_{j}\right\}$ is thin.

It is worth mentioning here that free interpolating sequences for the Nevanlinna class have been studied in [6].

It has been noticed that "thinness" of a sequence $\left\{z_{j}\right\}$ enables one to interpolate with functions that "oscillate little". A remark to that effect can be found in 10, p. 552, where the above observation is traced back to Jones' paper [7. The phenomenon also manifests itself clearly in Wolff's work [1] on the algebra $Q A:=$ $H^{\infty} \cap$ VMO. Among other things, it was proved in 11] that thin sequences are precisely the ones for which every interpolation problem (1.1), with $\left\{a_{j}\right\} \in \ell^{\infty}$, has a solution $f \in Q A$; see also 10 for an extension of this to general Douglas algebras. Our current results can be viewed as another illustration of the same principle. For example, Proposition 1.4 basically tells us that on thin sequences $\left\{z_{j}\right\}$ one can interpolate large values by functions in $\mathcal{N}$ that never become too small (and hence do not oscillate too much).

Now we go on to discuss some further restatements of the two equivalent conditions in Theorem 1.3. Having arisen in the course of the proof, these restatements are (hopefully) interesting in their own right; we have listed them in Theorem 1.5 and Proposition 1.6 below.

Theorem 1.5. Given an interpolating sequence $\left\{z_{j}\right\} \subset \mathbb{D}$, the following are equivalent:

(i) $\left\{z_{j}\right\}$ is thin.

(ii) There is a sequence $\left\{m_{j}\right\} \subset(0,1)$ with $\lim _{j \rightarrow \infty} m_{j}=1$ such that every (Nevanlinna-Pick) interpolation problem

$$
F\left(z_{j}\right)=w_{j} \quad \text { with } \quad\left|w_{j}\right| \leq m_{j} \quad(j=1,2, \ldots)
$$

has a solution $F \in H^{\infty}$ with $\|F\|_{\infty} \leq 1$.

(iii) $\left\{z_{j}\right\}$ is an NVI-sequence.

(iv) There is a sequence $\left\{M_{j}\right\} \subset(0, \infty)$ with $\lim _{j \rightarrow \infty} M_{j}=\infty$ such that every interpolation problem

$$
U\left(z_{j}\right)=u_{j} \quad \text { with } \quad 0 \leq u_{j} \leq M_{j} \quad(j=1,2, \ldots)
$$

can be solved with a harmonic function $U: \mathbb{D} \rightarrow \mathbb{R}$ satisfying $\inf \{U(z): z \in \mathbb{D}\}>$ $-\infty$.

It is well known that if $\left\{z_{j}\right\}$ is an interpolating sequence, then every interpolation problem (1.1) with $\sup _{j}\left|a_{j}\right| \leq 1$ admits a solution $f \in H^{\infty}$ satisfying $\|f\|_{\infty} \leq C$, where $C=C\left(\left\{z_{j}\right\}\right)$ is a suitable constant. It is not so clear why such an estimate should exist in the context of "nonvanishing interpolation", where the principles of linear analysis no longer apply. However, for NVI-sequences $\left\{z_{j}\right\}$, we do have some control over the norms of interpolating functions, provided that the minorant $\left\{\varepsilon_{j}\right\}$ is chosen appropriately. The resulting (formally stronger) version of the NVIproperty is dealt with in the proposition below, along with a similar refinement of property (iv) in Theorem 1.5 
Proposition 1.6. For an interpolating sequence $\left\{z_{j}\right\} \subset \mathbb{D}$, each of the following is equivalent to (any of) the conditions (i)-(iv) in Theorem 1.5:

(v) There exist a positive constant $M=M\left(\left\{z_{j}\right\}\right)$ and a minorant $\left\{\varepsilon_{j}\right\}$ such that, whenever (1.5) holds, the interpolation problem $f\left(z_{j}\right)=a_{j}(j=1,2, \ldots)$ has a nonvanishing solution $f \in H^{\infty}$ with $\|f\|_{\infty} \leq M$.

(vi) There is a sequence $\left\{N_{j}\right\} \subset(1, \infty)$ with $\lim _{j \rightarrow \infty} N_{j}=\infty$ such that every interpolation problem

$$
V\left(z_{j}\right)=v_{j} \quad \text { with } \quad 1 \leq v_{j} \leq N_{j} \quad(j=1,2, \ldots)
$$

can be solved with a positive harmonic function $V$ on $\mathbb{D}$.

We now point out several consequences of the (i) $\Longrightarrow$ (ii) part of Theorem 1.5. First we remark that the interpolating function in (ii), as constructed in the proof below, will actually be a Blaschke product with thin zero sequence. (This fact, though not really needed for our purposes, can be verified along the lines of Earl [3], whose method we borrow.) Therefore, our result extends an earlier theorem from [9, where interpolating - and thin - Blaschke products solving NevanlinnaPick problems were shown to exist under more restrictive hypotheses.

Another by-product of (the same part of) Theorem 1.5 is a new, simpler, proof of the following result on asymptotic interpolation that was recently obtained by Gorkin and Mortini; cf. Theorem 2.3 in [5].

Corollary 1.7. Given a thin sequence $\left\{z_{j}\right\} \subset \mathbb{D}$ and a data sequence $\left\{a_{j}\right\} \in \ell^{\infty}$ with $\sup _{j}\left|a_{j}\right| \leq 1$, one can find a function $F \in H^{\infty}$ with $\|F\|_{\infty} \leq 1$ for which $F\left(z_{j}\right)-a_{j} \rightarrow 0$.

To derive this from Theorem 1.5 put $w_{j}=r_{j} a_{j} /\left|a_{j}\right|$, where $r_{j}=\min \left(\left|a_{j}\right|, m_{j}\right)$ and the numbers $m_{j}$ are the same as in condition (ii) above. Since $\left|w_{j}\right| \leq m_{j}$ and $w_{j}-a_{j} \rightarrow 0$, the (i) $\Longrightarrow$ (ii) part of the theorem ensures that the Nevanlinna-Pick problem

$$
F\left(z_{j}\right)=w_{j} \quad(j=1,2, \ldots), \quad\|F\|_{\infty} \leq 1,
$$

has a solution $F \in H^{\infty}$, and this $F$ will satisfy the conclusion of Corollary 1.7

Using the terminology of [5], Corollary 1.7 can be rephrased by saying that every thin sequence is an asymptotic interpolating sequence of type 1 . (The converse is also established in [5.) The proof given in 5] involves maximal ideals and depends heavily on earlier work of Wolff; our method is more elementary.

As a final application of Theorem 1.5, we briefly discuss interpolation by analytic functions that take values in a prescribed hyperbolic domain $\Omega \subset \mathbb{C}$, possibly unbounded. (A hyperbolic domain $\Omega$ in $\mathbb{C}$ is, by definition, one whose complement $\mathbb{C} \backslash \Omega$ contains at least two points.) For such a domain, let $w_{0} \in \Omega$ be any of its points, and let $\Phi: \mathbb{D} \rightarrow \Omega$ be the analytic universal covering map with

$$
\Phi(0)=w_{0} \quad \text { and } \quad \Phi^{\prime}(0)>0 .
$$

The pseudohyperbolic distance in $\Omega$ is then defined by

$$
\rho_{\Omega}\left(w_{1}, w_{2}\right):=\inf \left\{\rho\left(z_{1}, z_{2}\right): z_{1} \in \Phi^{-1}\left(w_{1}\right), z_{2} \in \Phi^{-1}\left(w_{2}\right)\right\} .
$$

Corollary 1.8. Suppose $\left\{z_{j}\right\} \subset \mathbb{D}$ is a thin sequence, $\Omega$ is a hyperbolic domain in $\mathbb{C}$, and $w_{0} \in \Omega$. Then there is a sequence $\left\{m_{j}\right\} \subset(0,1)$ with $\lim _{j \rightarrow \infty} m_{j}=1$ such 
that every interpolation problem

$$
G\left(z_{j}\right)=w_{j} \quad \text { with } \quad w_{j} \in \Omega, \rho_{\Omega}\left(w_{0}, w_{j}\right)<m_{j} \quad(j=1,2, \ldots)
$$

can be solved with an analytic function $G: \mathbb{D} \rightarrow \Omega$.

This, again, is readily deduced from the "Nevanlinna-Pick part" of Theorem 1.5 . Indeed, let $m_{j}$ be the same as in statement (ii) of the theorem, and let $\Phi: \mathbb{D} \rightarrow \Omega$ be the universal covering map normalized by (1.8), as above. We know that, for each fixed $j \geq 1$, there are points $\zeta_{0}, \zeta_{j}$ in $\mathbb{D}$ satisfying $\Phi\left(\zeta_{0}\right)=w_{0}, \Phi\left(\zeta_{j}\right)=w_{j}$ and $\rho\left(\zeta_{0}, \zeta_{j}\right)<m_{j}$. In fact, we can take $\zeta_{0}=0$. (Otherwise, note that there is a conformal automorphism $\tau: \mathbb{D} \rightarrow \mathbb{D}$ with $\tau\left(\zeta_{0}\right)=0, \Phi \circ \tau=\Phi$ and replace the points $\zeta_{0}, \zeta_{j}$ by their $\tau$-images.) Thus we have $\left|\zeta_{j}\right|=\rho\left(0, \zeta_{j}\right)<m_{j}$, and Theorem 1.5 provides us with an analytic function $F: \mathbb{D} \rightarrow \mathbb{D}$ satisfying $F\left(z_{j}\right)=\zeta_{j}$ for all $j \geq 1$. The function $G=\Phi \circ F$ now has the required properties, and the result follows.

In particular, letting $\Omega=\mathbb{D} \backslash\{0\}$, we arrive at an amusing mixture of the nonvanishing interpolation problem and the Nevanlinna-Pick problem. In this case, the universal covering map (corresponding to the choice $w_{0}=1 / e$ ) is given by $\Phi(z)=\exp [(z-1) /(z+1)]$, so the distance $\rho_{\Omega}(\cdot, \cdot)$ is easily computable, and Corollary 1.8 provides an explicit solvability criterion.

Going back to Theorem 1.3 one might wish to obtain a more quantitative version thereof by characterizing the minorants $\left\{\varepsilon_{j}\right\}$ associated with a fixed NVI-sequence $\left\{z_{j}\right\}$. Equivalently, given a sequence $\left\{\varepsilon_{j}\right\} \subset(0,1)$ with $\lim _{j \rightarrow \infty} \varepsilon_{j}=0$, one might look for a characterization of those NVI-sequences $\left\{z_{j}\right\}$ for which $\left\{\varepsilon_{j}\right\}$ serves as a minorant. In fact, our proof of Theorem 1.3 (or rather of its extended version, Theorem 1.5) does yield some estimates of the quantities $\varepsilon_{j}$ and $1-\delta_{j}$, where

$$
\delta_{j}:=\prod_{k: k \neq j}\left|\frac{z_{j}-z_{k}}{1-\bar{z}_{j} z_{k}}\right|,
$$

in terms of each other. However, there is a gap between the estimates coming from the "if" and "only if" parts of the proof (i.e., from the proofs of implications (i) $\Longrightarrow$ (iii) and (iii) $\Longrightarrow$ (i) in Theorem 1.5). We now restrict ourselves to a specific class of minorants, which enables us to state the quantitative result in an "if and only if" form.

Theorem 1.9. Let $\left\{z_{j}\right\}$ be an NVI-sequence in $\mathbb{D}$. The following are equivalent:

(a) For every $\alpha>0$, the sequence $\left\{\exp \left(-j^{\alpha}\right)\right\}$ is a minorant for $\left\{z_{j}\right\}$.

(b) For every $\beta>0$, we have

$$
1-\inf _{k: k \neq j} \rho\left(z_{j}, z_{k}\right)=O\left(j^{-\beta}\right) \quad \text { as } \quad j \rightarrow \infty .
$$

Unlike the qualitative results above, Theorem 1.9 will be established via the classical Nevanlinna-Pick theorem by checking that a certain matrix is positive definite. A similar technique was recently used by Koosis [8] in connection with Carleson's interpolation theorem.

The rest of the paper mainly deals with the proofs. These are preceded by some preparatory lemmas and followed by a list of open questions that puzzle us. The lemmas are collected in Section 2, the proofs of our main results are given in Sections 3 and 4, and the questions are posed in Section 5 .

The authors thank Joaquim Bruna for helpful discussions. 


\section{Some Lemmas}

We begin by introducing some (standard) notation.

Let $I$ be an arc of the unit circle $\mathbb{T}:=\partial \mathbb{D}$. We write $m(I)$ for the normalized length of $I$ (so that $m(\mathbb{T})=1$ ) and, given a number $N \geq 1$, we let $N I$ denote the arc $J \subset \mathbb{T}$ which has the same midpoint as $I$ and satisfies $m(J)=N m(I)$. (In case $N m(I) \geq 1$, it is understood that $N I=\mathbb{T}$.) Further, $S(I)$ will stand for the "Carleson box" with base $I$; that is,

$$
S(I):=\left\{z \in \mathbb{D} \backslash\{0\}: \frac{z}{|z|} \in I,|z|>1-m(I)\right\} .
$$

Finally, to a point $z \in \mathbb{D} \backslash\{0\}$ we associate the arc $I_{z}$ with midpoint $z /|z|$ and length $m\left(I_{z}\right)=1-|z|$; and if $z=0$, then we set $I_{z}=\mathbb{T}$.

The following characterization of thin sequences is due to Sundberg and Wolff. It can be obtained by combining Lemma 7.1 on p. 578 of 10 with the concluding paragraph on p. 580 that follows the lemma's proof. The underlying Douglas algebra $B$ that figures in [10] is here taken to be $H^{\infty}+C$.

Lemma 2.1. A sequence of distinct points $\left\{z_{j}\right\} \subset \mathbb{D}$ is thin if and only if, for every $N \geq 1$,

$$
\lim _{j \rightarrow \infty}\left(1-\left|z_{j}\right|\right)^{-1} \sum_{k \in \mathcal{K}(N, j)}\left(1-\left|z_{k}\right|\right)=0
$$

where $\mathcal{K}(N, j)$ is the set of those $k \in \mathbb{N} \backslash\{j\}$ for which $z_{k} \in S\left(N I_{z_{j}}\right)$.

The next lemma is also borrowed from [10] (see Lemma 5.4 on p. 573 of that paper; put $B=H^{\infty}+C$ to arrive at the version below).

Lemma 2.2. Suppose $\left\{z_{j}\right\} \subset \mathbb{D}$ is a thin sequence. Then there are numbers $\tau_{j} \in$ $(0,1), \gamma_{j} \in(0,1)$ with $\tau_{j} \rightarrow 1$ and $\gamma_{j} \rightarrow 1$ such that whenever $\left\{\zeta_{j}\right\}$ is a sequence in $\mathbb{D}$ satisfying

it follows that

$$
\rho\left(\zeta_{j}, z_{j}\right)<\tau_{j} \quad(j=1,2, \ldots),
$$

$$
\prod_{k: k \neq j} \rho\left(\zeta_{j}, \zeta_{k}\right) \geq \gamma_{j} \quad(j=1,2, \ldots) .
$$

The following result is an adaptation of J.P. Earl's key lemma on which his interpolation method is based; see Lemma 4 in [3] or Lemma 5.4 in [4, Chapter VII.

Lemma 2.3. Given $n \in \mathbb{N}$, suppose $z_{1}, \ldots, z_{n}$ are distinct points in $\mathbb{D}$ and $\tau_{1}, \ldots, \tau_{n}$ are numbers in $(0,1)$ such that the disks

$$
\Delta_{j}:=\left\{\zeta \in \mathbb{D}: \rho\left(\zeta, z_{j}\right) \leq \tau_{j}\right\} \quad(j=1, \ldots, n)
$$

are pairwise disjoint. Further, put

$$
\mu_{j}^{(n)}:=\inf \left\{\prod_{1 \leq k \leq n, k \neq j} \rho\left(z_{j}, \zeta_{k}\right): \zeta_{1} \in \Delta_{1}, \ldots, \zeta_{n} \in \Delta_{n}\right\}
$$

and assume that $w_{1}, \ldots, w_{n}$ are complex numbers with

$$
\left|w_{j}\right| \leq \tau_{j} \mu_{j}^{(n)} \quad(j=1, \ldots, n) .
$$


Then there are points $\zeta_{1} \in \Delta_{1}, \ldots, \zeta_{n} \in \Delta_{n}$ such that the Blaschke product

$$
B_{\zeta_{1}, \ldots, \zeta_{n}}(z):=\prod_{k=1}^{n} \frac{z-\zeta_{k}}{1-\bar{\zeta}_{k} z} \cdot \frac{1-\bar{\zeta}_{k}}{1-\zeta_{k}}
$$

satisfies

$$
B_{\zeta_{1}, \ldots, \zeta_{n}}\left(z_{j}\right)=w_{j} \quad(j=1, \ldots, n) .
$$

The only difference between Earl's original version and ours, as stated above, is that in [3] the noneuclidean radii $\tau_{j}$ of the disks involved are all equal to a single constant $\tau$. However, Earl's proof works in our situation as well, once obvious adjustments are made.

Lemma 2.4. Let $z \in \mathbb{D}, M>0$, and let $V$ be a positive harmonic function on $\mathbb{D}$. Suppose $z_{1}, \ldots, z_{m}$ are points lying in $S\left(I_{z}\right)$ with the properties that

$$
V\left(z_{k}\right) \geq M \quad(k=1, \ldots, m)
$$

and

$$
\delta:=\min _{1 \leq k \leq m} \prod_{l: l \neq k} \rho\left(z_{k}, z_{l}\right)>0 .
$$

Then

$$
V(z) \geq c(\delta) \frac{M}{1-|z|} \sum_{k=1}^{m}\left(1-\left|z_{k}\right|\right)
$$

where $c(\delta)$ is a positive constant depending only on $\delta$.

Proof. Replacing $V$ by $V \circ \tau$, where $\tau$ is the conformal automorphism of the disk which interchanges $z$ and 0 , we may assume that $z=0$. Also, there is no loss of generality in assuming that $V$ is continuous on $\mathbb{D} \cup \mathbb{T}$.

For each $n=0,1,2, \ldots$, let us partition the circle $\mathbb{T}$ into $2^{n}$ dyadic arcs

$$
I_{n, k}=\left\{e^{i \theta}: 2 \pi(k-1) 2^{-n} \leq \theta<2 \pi k \cdot 2^{-n}\right\} \quad\left(k=1, \ldots, 2^{n}\right) .
$$

This done, consider the Carleson boxes $Q_{n, k}:=S\left(I_{n, k}\right)$; these will be referred to as "dyadic squares". Further, let $T\left(Q_{n, k}\right)$ denote the "top half" of $Q_{n, k}$, that is,

$$
T\left(Q_{n, k}\right)=\left\{z \in Q_{n, k}:|z| \leq 1-2^{-n-1}\right\} .
$$

Now we use a stopping time argument. Namely, among the squares $Q_{n, k}$ we select the maximal ones, say $Q^{j}$, whose top halves hit the set $\mathcal{Z}:=\left\{z_{1}, \ldots, z_{m}\right\}$. Thus we obtain finitely many dyadic squares $Q^{1}, \ldots, Q^{N}$, each of which satisfies $T\left(Q^{j}\right) \cap \mathcal{Z} \neq$ $\emptyset$ and is contained in no larger dyadic square $Q$ with $T(Q) \cap \mathcal{Z} \neq \emptyset$. We then have $\mathcal{Z} \subset Q^{1} \cup \cdots \cup Q^{N}$. Also, writing $I^{j}$ for the base (i.e., radial projection) of $Q^{j}$, we observe that the $\operatorname{arcs} I^{j}$ are pairwise disjoint, as are the squares $Q^{j}$ themselves. Furthermore, (2.4) implies the Carleson measure estimate

$$
\sum_{z_{l} \in Q^{j}}\left(1-\left|z_{l}\right|\right) \leq C(\delta) \cdot m\left(I^{j}\right) \quad(j=1, \ldots, N),
$$

where $C(\delta)$ is a constant depending only on $\delta$. Summing over $j$, we deduce from the preceding inequality that

$$
\sum_{l=1}^{m}\left(1-\left|z_{l}\right|\right) \leq C(\delta) \sum_{j=1}^{N} m\left(I^{j}\right) .
$$


By construction, $T\left(Q^{j}\right)$ contains a point of $\mathcal{Z}$; renumbering the points, if necessary, we may call this point $z_{j}$. Now let $L_{j}$ be the closed "top side" of $Q^{j}$, defined by

$$
L_{j}:=\left\{\left(1-m\left(I^{j}\right)\right) e^{i \theta}: e^{i \theta} \in \operatorname{clos} I^{j}\right\},
$$

and consider the domain $\Omega:=\mathbb{D} \backslash \bigcup_{j=1}^{N} L_{j}$. We claim that there is an absolute constant $c_{1}>0$ such that

$$
V(\zeta) \geq c_{1} \sum_{j=1}^{N} V\left(z_{j}\right) \omega\left(\zeta, L_{j}, \Omega\right), \quad \zeta \in \Omega,
$$

where $\omega(\cdot, E, \Omega)$ denotes the harmonic measure of the set $E \subset \partial \Omega$ with respect to $\Omega$.

Indeed, by the maximum principle, it suffices to verify (2.6) for $\zeta \in \partial \Omega=$ $\mathbb{T} \cup \bigcup_{j=1}^{N} L_{j}$. Now if $\zeta \in L_{j}$ for some $j$, then (2.6) reduces to $V(\zeta) \geq c_{1} V\left(z_{j}\right)$, which holds with a suitable $c_{1}$ by virtue of Harnack's inequality (note that $\rho\left(\zeta, z_{j}\right) \leq c_{2}<$ 1 with an absolute constant $c_{2}$ ). And if $\zeta \in \mathbb{T}$, then (2.6) is obvious, since the right-hand side equals 0 .

Applying (2.6) with $\zeta=0$ and using the assumption (2.3), we get

$$
V(0) \geq c_{1} M \omega\left(0, \bigcup_{j=1}^{N} L_{j}, \Omega\right) .
$$

Since the radial projection of $\bigcup_{j=1}^{N} L_{j}$ is clos $\left(\bigcup_{j=1}^{N} I^{j}\right)$, the $\operatorname{arcs} I^{j}$ being pairwise disjoint, Hall's lemma yields

$$
\omega\left(0, \bigcup_{j=1}^{N} L_{j}, \Omega\right) \geq c_{3} \sum_{j=1}^{N} m\left(I^{j}\right)
$$

with some numerical constant $c_{3}>0$. Combining this with (2.7) and (2.5), we obtain

as required.

$$
V(0) \geq c(\delta) M \sum_{k=1}^{m}\left(1-\left|z_{k}\right|\right)
$$

\section{Proofs of Theorem 1.5 and Proposition 1.6}

Proof of Theorem 1.5. (i) $\Longrightarrow$ (ii). Let $\tau_{j}$ and $\gamma_{j}$ (with $j=1,2, \ldots$ ) be the parameters associated to $\left\{z_{j}\right\}$ as in Lemma 2.2. Put

$$
m_{j}:=\tau_{j} \gamma_{j} \quad(j=1,2, \ldots)
$$

and note that $0<m_{j}<1$ for all $j$, while $\lim _{j \rightarrow \infty} m_{j}=1$.

Now if $\left\{w_{j}\right\}$ is a sequence of complex numbers with $\left|w_{j}\right| \leq m_{j}$, then, for any fixed $n \in \mathbb{N}$, we have (2.2), since

$$
\gamma_{j} \leq \mu_{j}^{(n)} \quad(j=1, \ldots, n) .
$$

Here, the numbers $\mu_{j}^{(n)}$ are defined by (2.1), where the disks $\Delta_{j}$ have our current $\tau_{j}$ 's (the ones coming from Lemma 2.2) as their noneuclidean radii. The inequality (3.2) is then immediate, because the products appearing in (2.1) are all $\geq \gamma_{j}$ by Lemma 2.2 
Now Lemma 2.3 tells us that every finite interpolation problem

$$
F\left(z_{j}\right)=w_{j} \quad(j=1, \ldots, n)
$$

can be solved with a finite Blaschke product (which is, of course, an $H^{\infty}$-function of norm 1). By normal families, the infinite interpolation problem

$$
F\left(z_{j}\right)=w_{j} \quad(j=1,2, \ldots)
$$

can also be solved with a unit-norm function $F \in H^{\infty}$.

(ii) $\Longrightarrow$ (iii). Put

$$
\varepsilon_{j}:=\exp \left(-\frac{m_{j}}{1-m_{j}}\right) \quad(j=1,2, \ldots),
$$

where $m_{j}$ are the numbers that figure in (ii). Since $0<m_{j}<1$ and $m_{j} \rightarrow 1$, we have $0<\varepsilon_{j}<1$ and $\varepsilon_{j} \rightarrow 0$. We now let $\left\{a_{j}\right\}$ be a sequence of complex numbers with

$$
1 \geq\left|a_{j}\right| \geq \varepsilon_{j} \quad(j=1,2, \ldots),
$$

and we want to find a nonvanishing function $f \in H^{\infty}$ that interpolates $a_{j}$ at $z_{j}$. This will be constructed in the form

$$
f(z)=\exp \left\{\frac{g(z)}{g(z)-1}+i h(z)\right\}, \quad z \in \mathbb{D},
$$

where $g$ and $h$ are $H^{\infty}$-functions that satisfy

$$
\frac{g\left(z_{j}\right)}{g\left(z_{j}\right)-1}=\log \left|a_{j}\right| \quad(j=1,2, \ldots), \quad\|g\|_{\infty} \leq 1,
$$

and

$$
h\left(z_{j}\right)=\arg a_{j} \quad(j=1,2, \ldots) .
$$

Here, it is understood that $\arg (\cdot)$ takes values in $(-\pi, \pi]$. In particular, (3.7) indeed has a solution $h \in H^{\infty}$, since $\left\{\arg a_{j}\right\} \in \ell^{\infty}$ and $\left\{z_{j}\right\}$ is an interpolating sequence.

To see that (3.6) can be solved with a function $g \in H^{\infty}$, we rewrite it as

$$
g\left(z_{j}\right)=w_{j} \quad(j=1,2, \ldots), \quad\|g\|_{\infty} \leq 1,
$$

where

$$
w_{j}:=\frac{\log \left|a_{j}\right|}{\log \left|a_{j}\right|-1}=1-\left(1+\log \frac{1}{\left|a_{j}\right|}\right)^{-1} .
$$

From (3.3) and (3.4) it follows that

$$
0 \leq w_{j} \leq 1-\left(1+\log \frac{1}{\varepsilon_{j}}\right)^{-1}=m_{j}
$$

so the solvability of the Nevanlinna-Pick problem (3.8) is guaranteed by (ii).

The function $f$ given by (3.5) is then analytic and zero-free in $\mathbb{D}$, and it satisfies $f\left(z_{j}\right)=a_{j}$ by virtue of (3.6) and (3.7). Finally, since the image of $\mathbb{D}$ under the mapping $w(\zeta)=\zeta /(\zeta-1)$ is the half-plane $\mathcal{H}:=\left\{\operatorname{Re} w<\frac{1}{2}\right\}$, we conclude (even though it remains unclear whether $\partial \mathcal{H}$ contains the nontrivial zeros of the Riemann zeta-function) that

$$
\operatorname{Re}\left\{\frac{g(z)}{g(z)-1}\right\} \leq \frac{1}{2}, \quad z \in \mathbb{D}
$$


and so $f$ is bounded with

$$
\|f\|_{\infty} \leq \exp \left(\frac{1}{2}+\|h\|_{\infty}\right)
$$

Therefore, $\left\{\varepsilon_{j}\right\}$ is a minorant for $\left\{z_{j}\right\}$, and $\left\{z_{j}\right\}$ is an NVI-sequence.

(iii) $\Longrightarrow$ (iv). Given a minorant $\left\{\varepsilon_{j}\right\}$ associated with $\left\{z_{j}\right\}$, put $M_{j}:=\log \left(1 / \varepsilon_{j}\right)$ and suppose $\left\{u_{j}\right\}$ is a sequence of numbers with $0 \leq u_{j} \leq M_{j}$. Letting $a_{j}:=$ $\exp \left(-u_{j}\right)$, we then have $1 \geq a_{j} \geq \varepsilon_{j}$, and condition (iii) ensures that there is a nonvanishing function $f \in H^{\infty}$ with $f\left(z_{j}\right)=a_{j}$. It remains to notice that

$$
U(z):=\log \frac{1}{|f(z)|}, \quad z \in \mathbb{D}
$$

is a harmonic function which interpolates $u_{j}$ at $z_{j}$ and which is bounded from below by $-\log \|f\|_{\infty}$.

(iv) $\Longrightarrow$ (i). Suppose $\left\{z_{j}\right\}$ fails to be thin. Then, by Lemma 2.1, there exist numbers $N \geq 1, \varepsilon>0$ and an infinite set of indices $\mathcal{J} \subset \mathbb{N}$ such that

$$
\sum_{k \in \mathcal{K}(N, j)}\left(1-\left|z_{k}\right|\right)>\varepsilon\left(1-\left|z_{j}\right|\right) \text { for all } j \in \mathcal{J}
$$

where $\mathcal{K}(N, j)$ has the same meaning as in Lemma 2.1 It follows that each $\mathcal{K}(N, j)$ contains a finite subset $\mathcal{F}(N, j)$ such that

$$
\sum_{k \in \mathcal{F}(N, j)}\left(1-\left|z_{k}\right|\right)>\varepsilon\left(1-\left|z_{j}\right|\right) \quad \text { for all } \quad j \in \mathcal{J} .
$$

We note, in particular, that $\mathcal{F}(N, j) \subset \mathbb{N} \backslash\{j\}$ and that $z_{k} \in S\left(N I_{z_{j}}\right)$ if $k \in \mathcal{F}(N, j)$ (recall the definition of $\mathcal{K}(N, j)$ in Lemma 2.1). Furthermore, replacing $\mathcal{J}$ by a sparser subset if necessary, we may assume that $\mathcal{F}(N, j) \cap \mathcal{J}=\emptyset$ for all $j \in \mathcal{J}$.

Now let $U$ be a (real-valued) harmonic function satisfying $U\left(z_{j}\right)=0$ for $j \in \mathcal{J}$, $U\left(z_{k}\right)=M_{k}$ for $k \notin \mathcal{J}$ and

$$
U(z) \geq-A, \quad z \in \mathbb{D},
$$

with some constant $A>0$. (Here, $M_{j}$ are the numbers figuring in (iv), and the latter condition ensures the existence of a function $U$ with the above properties.)

Consider the positive harmonic function

$$
V(z):=U(z)+A, \quad z \in \mathbb{D} .
$$

Next, fix a point $z_{j}$ with $j \in \mathcal{J}$, where $j$ is large enough in order that $1-\left|z_{j}\right|<N^{-1}$, and let $w_{j}=w_{N, j}$ denote the point of the segment $\left[0, z_{j}\right]$ for which

$$
1-\left|w_{j}\right|=N\left(1-\left|z_{j}\right|\right) .
$$

It is easy to check that

$$
\rho\left(z_{j}, w_{j}\right) \leq 1-\frac{1}{N},
$$

and so Harnack's inequality tells us that

$$
V\left(w_{j}\right) \leq \frac{1+\rho\left(z_{j}, w_{j}\right)}{1-\rho\left(z_{j}, w_{j}\right)} V\left(z_{j}\right) \leq(2 N-1) A .
$$

On the other hand, for $k \in \mathcal{F}(N, j)$ we have

$$
V\left(z_{k}\right)=M_{k}+A \geq M_{j}^{*}+A,
$$


where

$$
M_{j}^{*}:=\min \left\{M_{k}: k \in \mathcal{F}(N, j)\right\} .
$$

Consequently, invoking Lemma 2.4 and then inequality (3.10), we obtain

$$
V\left(w_{j}\right) \geq c(\delta) \frac{M_{j}^{*}+A}{N\left(1-\left|z_{j}\right|\right)} \sum_{k \in \mathcal{F}(N, j)}\left(1-\left|z_{k}\right|\right) \geq c(\delta) \frac{\varepsilon}{N}\left(M_{j}^{*}+A\right) .
$$

It is clear that $M_{j}^{*} \rightarrow \infty$ as $j \rightarrow \infty, j \in \mathcal{J}$, and so (3.12) yields

$$
V\left(w_{j}\right) \rightarrow \infty \quad \text { as } \quad j \rightarrow \infty, j \in \mathcal{J} .
$$

This contradicts (3.11) and completes the proof.

Proof of Proposition 1.6. Now that Theorem 1.5 is proved, it suffices to show that (i) $\Longrightarrow$ (v) $\Longrightarrow$ (vi) $\Longrightarrow$ (iv).

The first of these implications is actually hidden in the (i) $\Longrightarrow$ (ii) $\Longrightarrow$ (iii) parts of the preceding proof. Indeed, if (i) holds, and if $m_{j}$ and $\varepsilon_{j}$ are defined by (3.1) and (3.3) respectively, then our interpolation problem admits a solution of the form (3.5), which satisfies (3.9). Also, since $h$ is only required to be an $H^{\infty}$-function solving (3.7), we are free to assume that $\|h\|_{\infty} \leq C(\delta)$, where $\delta=\delta\left(\left\{z_{j}\right\}\right)$ is the value of the infimum in (1.2) and $C(\delta)$ is an appropriate constant depending only on $\delta$ (see [4, Chapter VII). Consequently, (3.9) yields

$$
\|f\|_{\infty} \leq \exp \left(\frac{1}{2}+C(\delta)\right)
$$

and this last quantity is eligible as $M\left(\left\{z_{j}\right\}\right)$ in (v).

To see that (v) implies (vi), define

$$
N_{j}:=1-\frac{\log \varepsilon_{j}}{\log M} \quad(j=1,2, \ldots),
$$

where $M=M\left(\left\{z_{j}\right\}\right)$ and $\varepsilon_{j}$ have the same meaning as in (v). (In particular, one has $M>1$.) Next, given a sequence of values $\left\{v_{j}\right\}$ with $1 \leq v_{j} \leq N_{j}$, put $a_{j}:=M^{1-v_{j}}$ and note that $\varepsilon_{j} \leq a_{j} \leq 1$; then use (v) to produce a nonvanishing function $f \in H^{\infty}$ such that $f\left(z_{j}\right)=a_{j}$ (for all $j$ ) and $\|f\|_{\infty} \leq M$. This done, observe that

$$
V(z):=1-\frac{\log |f(z)|}{\log M}
$$

is a positive harmonic function on $\mathbb{D}$ and

$$
V\left(z_{j}\right)=1-\frac{\log a_{j}}{\log M}=v_{j} \quad(j=1,2, \ldots) .
$$

The remaining implication (vi) $\Longrightarrow$ (iv) is immediate: once (vi) holds, we put $M_{j}:=N_{j}-1$ and arrive at (iv) with $U=V-1$, where $V$ is a positive harmonic function that interpolates $u_{j}+1$ at $z_{j}$.

\section{Proof of Theorem 1.9}

(a) $\Longrightarrow$ (b). Fix $\alpha>0$, and put

$$
\varepsilon_{j}:=\exp \left(-j^{\alpha}\right), \quad \rho_{j}:=\inf _{k: k \neq j} \rho\left(z_{j}, z_{k}\right) .
$$


Since $\rho_{j}<1$, we have $\rho_{j}<\frac{1}{2}\left(1+\rho_{j}\right)$. Consequently, for each $j \in \mathbb{N}$ there exists an index $k=k(j) \in \mathbb{N} \backslash\{j\}$ such that

$$
\rho\left(z_{j}, z_{k}\right)<\frac{1}{2}\left(1+\rho_{j}\right)
$$

or, equivalently,

$$
1-\rho\left(z_{j}, z_{k}\right)>\frac{1}{2}\left(1-\rho_{j}\right)
$$

Now suppose we can find an infinite set of indices $\mathcal{J} \subset \mathbb{N}$ with the property that

$$
j^{\alpha}\left(1-\rho_{j}\right) \rightarrow \infty \quad \text { as } \quad j \rightarrow \infty, j \in \mathcal{J} .
$$

Replacing $\mathcal{J}$ by a suitable subset thereof, we may further assume that $k(j) \notin \mathcal{J}$ for all $j \in \mathcal{J}$.

Since $\left\{\varepsilon_{j}\right\}$ is a minorant for $\left\{z_{j}\right\}$, there is a nonvanishing function $f \in H^{\infty}$ such that $f\left(z_{j}\right)=\varepsilon_{j}$ for all $j \in \mathcal{J}$ and $f\left(z_{l}\right)=1$ for all $l \in \mathbb{N} \backslash \mathcal{J}$. Harnack's inequality, applied to the positive harmonic function $z \mapsto \log \left(\|f\|_{\infty} /|f(z)|\right)$, now yields

$$
\frac{\log \left(\|f\|_{\infty} / \varepsilon_{j}\right)}{\log \|f\|_{\infty}} \leq \frac{1+\rho\left(z_{j}, z_{k}\right)}{1-\rho\left(z_{j}, z_{k}\right)} \quad(j \in \mathcal{J}, k=k(j))
$$

(we have used the second inequality in (1.6) with $a_{j}=\varepsilon_{j}$ and $a_{k}=1$ ). Noting that $\|f\|_{\infty}>1$ and

$$
\frac{1+\rho\left(z_{j}, z_{k}\right)}{1-\rho\left(z_{j}, z_{k}\right)} \leq \frac{4}{1-\rho_{j}}, \quad k=k(j)
$$

(this relies on (4.2)), we deduce from (4.4) that

$$
\log \frac{1}{\varepsilon_{j}} \leq \frac{4 \log \|f\|_{\infty}}{1-\rho_{j}}, \quad j \in \mathcal{J} .
$$

This in turn reduces to

$$
j^{\alpha}\left(1-\rho_{j}\right) \leq 4 \log \|f\|_{\infty}, \quad j \in \mathcal{J},
$$

which contradicts (4.3). The contradiction shows that

$$
1-\rho_{j}=O\left(j^{-\alpha}\right), \quad j \in \mathbb{N},
$$

and proves (b), since (4.5) has actually been verified for any $\alpha>0$.

(b) $\Longrightarrow(\mathrm{a})$. Fix $\alpha>0$, and suppose $\left\{a_{j}\right\}$ is a sequence of complex numbers with

$$
1 \geq\left|a_{j}\right| \geq \varepsilon_{j} \quad(j=1,2, \ldots),
$$

where $\varepsilon_{j}$ is defined as in (4.1). Then put

$$
w_{j}:=\log \frac{2}{a_{j}}=\log \frac{2}{\left|a_{j}\right|}+i \arg \frac{2}{a_{j}},
$$

where $\arg (\cdot)$ takes values in $(-\pi, \pi]$.

Assuming that condition (b) holds, we wish to find a nonvanishing function $f \in H^{\infty}$ satisfying

$$
f\left(z_{j}\right)=a_{j} \quad(j=1,2, \ldots) .
$$

We claim that this task will be accomplished if we check that, for some $N \in \mathbb{N}$, the matrix

$$
\mathcal{A}=\mathcal{A}_{N}:=\left\{\frac{w_{j}+\bar{w}_{k}}{1-\bar{z}_{j} z_{k}}\right\}_{j, k \geq N}
$$


is positive definite, in the sense that $\langle\mathcal{A} x, x\rangle \geq 0$ whenever $x=\left\{x_{j}\right\}_{j \geq N} \in \ell_{0}^{2}$. (Here $\ell_{0}^{2}$ is the set of $\ell^{2}$-sequences that have finitely many nonzero components, and $\langle\cdot, \cdot\rangle$ is the usual inner product in $\ell^{2}$.) Indeed, once $\mathcal{A}$ is known to be positive definite, the Nevanlinna-Pick theorem provides us with an analytic function $g$ on $\mathbb{D}$ satisfying $\operatorname{Re} g \geq 0$ and

$$
g\left(z_{j}\right)=w_{j} \quad(j \geq N) .
$$

Now let $b$ stand for the Blaschke product with zeros $\left\{z_{j}\right\}_{j \geq N}$, and let $h \in H^{\infty}$ be a solution to the finite interpolation problem

$$
h\left(z_{j}\right)=\frac{w_{j}-g\left(z_{j}\right)}{b\left(z_{j}\right)} \quad(j=1, \ldots, N-1) .
$$

Then $\phi:=g+b h$ is an analytic function on $\mathbb{D}$ with the properties that

$$
\operatorname{Re} \phi(z) \geq-\|h\|_{\infty}, \quad z \in \mathbb{D},
$$

and

$$
\phi\left(z_{j}\right)=w_{j} \quad \text { for all } j \in \mathbb{N} \text {. }
$$

Consequently, $f=2 \exp (-\phi)$ is a nonvanishing $H^{\infty}$-function that solves (4.6), and we are done.

Next we remark that the matrix $\mathcal{A}$ is (or is not) positive definite simultaneously with $\mathcal{B}=\mathcal{B}_{N}=\left\{B_{j k}\right\}_{j, k \geq N}$, where

$$
B_{j k}:=\frac{w_{j}+\bar{w}_{k}}{1-\bar{z}_{j} z_{k}}\left(1-\left|z_{j}\right|^{2}\right)^{1 / 2}\left(1-\left|z_{k}\right|^{2}\right)^{1 / 2} .
$$

Our aim is thus to prove that $\mathcal{B}$ is positive definite for a suitable choice of $N$. To this end, we write $\mathcal{B}=\mathcal{D}+\mathcal{M}$, where $\mathcal{D}$ is the diagonal part of $\mathcal{B}$ (i.e., the matrix obtained from $\mathcal{B}$ by replacing its off-diagonal entries with 0 ) and $\mathcal{M}:=\mathcal{B}-\mathcal{D}$ is the complementary matrix with zero diagonal. Now if $x=\left\{x_{j}\right\}_{j \geq N} \in \ell_{0}^{2}$, then

$$
\langle\mathcal{B} x, x\rangle=\langle\mathcal{D} x, x\rangle+\langle\mathcal{M} x, x\rangle \geq \sum_{j \geq N} B_{j j}\left|x_{j}\right|^{2}-\|\mathcal{M}\| \sum_{j \geq N}\left|x_{j}\right|^{2},
$$

where $\|\mathcal{M}\|=\|\mathcal{M}\|_{\ell^{2} \rightarrow \ell^{2}}$. Therefore, to show that $\mathcal{B}$ is positive definite, it is enough to verify that

$$
\|\mathcal{M}\| \leq \inf _{j \geq N} B_{j j}
$$

Also, since

$$
B_{j j}=2 \operatorname{Re} w_{j}=2 \log \frac{2}{\left|a_{j}\right|} \geq 2 \log 2>1,
$$

it will suffice to check that

$$
\|\mathcal{M}\| \leq 1
$$

once the parameter $N$ is chosen appropriately.

Using the Hilbert-Schmidt norm of the (self-adjoint) matrix $\mathcal{M}$ as a bound for $\|\mathcal{M}\|$, we get

$$
\|\mathcal{M}\|^{2} \leq \sum_{j, k \geq N, j \neq k}\left|B_{j k}\right|^{2}=2 \sum_{j=N+1}^{\infty} \sum_{k=N}^{j-1}\left|B_{j k}\right|^{2} .
$$

We further observe that

$$
\left|B_{j k}\right|^{2}=\frac{\left(1-\left|z_{j}\right|^{2}\right)\left(1-\left|z_{k}\right|^{2}\right)}{\left|1-\bar{z}_{j} z_{k}\right|^{2}}\left|w_{j}+\bar{w}_{k}\right|^{2}=\left(1-\rho\left(z_{j}, z_{k}\right)^{2}\right)\left|w_{j}+\bar{w}_{k}\right|^{2} .
$$


An application of (b) with $\beta=2 \alpha+3$ now gives

$$
1-\rho\left(z_{j}, z_{k}\right)^{2} \leq 1-\rho_{j}^{2} \leq 2\left(1-\rho_{j}\right) \leq A j^{-2 \alpha-3} \quad(k \neq j)
$$

for some $A=A(\alpha)>0$. At the same time, the estimate

$$
\left|w_{j}\right| \leq C \log \frac{2}{\left|a_{j}\right|} \leq C \log \frac{2}{\varepsilon_{j}} \leq 2 C j^{\alpha}
$$

(which holds for all $j$ and some absolute $C>0$ ) tells us that

$$
\left|w_{j}+\bar{w}_{k}\right| \leq\left|w_{j}\right|+\left|w_{k}\right| \leq 4 C j^{\alpha} \quad(k<j) .
$$

With (4.10) and (4.11) plugged in, (4.9) yields

$$
\left|B_{j k}\right|^{2} \leq 16 A C^{2} j^{-2 \alpha-3} j^{2 \alpha}=\mathrm{const} \cdot j^{-3} \quad(k<j),
$$

and so

$$
\sum_{k=N}^{j-1}\left|B_{j k}\right|^{2} \leq \mathrm{const} \cdot(j-N) j^{-3} \leq \mathrm{const} \cdot j^{-2} \quad(j>N) .
$$

Finally, we go back to (4.8) and use (4.12) to obtain

$$
\|\mathcal{M}\|^{2} \leq \text { const } \sum_{j=N+1}^{\infty} \frac{1}{j^{2}} \leq \frac{\text { const }}{N} .
$$

Hence, taking $N$ to be appropriately large, we arrive at (4.7) and thereby complete the proof.

\section{Open QUestions}

Question 5.1. What is the quantitative description of the minorants $\left\{\varepsilon_{j}\right\}$ associated with a given thin sequence $\left\{z_{j}\right\}$ ? (We have already mentioned this problem in Section 1.) It would be nice to have the answer stated in terms of the "thinness parameters" (1.9).

Question 5.2. What happens to our free interpolation problem if nonvanishing functions are replaced by outer ones? Specifically, is it true that for each thin sequence $\left\{z_{j}\right\}$ there exists a minorant $\left\{\varepsilon_{j}\right\}$ such that every interpolation problem

$$
f\left(z_{j}\right)=a_{j} \quad \text { with } \quad 1 \geq\left|a_{j}\right| \geq \varepsilon_{j} \quad(j=1,2, \ldots)
$$

can be solved with an outer function $f \in H^{\infty}$ ?

Question 5.3. Consider the equivalence relation (i) $\Longleftrightarrow$ (iv) in Theorem 1.5 Does this generalize to higher dimensions (in several real variables), once thin sequences are properly defined?

To be more precise, let us begin by recalling that a sequence $\left\{z_{j}=\left(x_{j}, y_{j}\right)\right\}$ in the upper half-space

$$
\mathbb{R}_{+}^{n+1}=\left\{(x, y): x \in \mathbb{R}^{n}, y>0\right\}
$$

is said to be an interpolating sequence (for the class $h^{\infty}$ of bounded harmonic functions on $\left.\mathbb{R}_{+}^{n+1}\right)$ if every interpolation problem $u\left(z_{j}\right)=a_{j}(j=1,2, \ldots)$, with 
$\left\{a_{j}\right\} \in \ell^{\infty}$, has a solution $u \in h^{\infty}$. The problem of characterizing such sequences is still open (to the best of our knowledge) for $n>1$; see [1], 2] for some partial results on this matter.

We wish, however, to single out the class of those interpolating sequences $\left\{z_{j}\right\} \subset$ $\mathbb{R}_{+}^{n+1}$ which have the following property, referred to hereafter as $(*)$ : For some "majorant" $\left\{M_{j}\right\} \subset(1, \infty)$ satisfying $\lim _{j \rightarrow \infty} M_{j}=\infty$, every interpolation problem

$$
U\left(z_{j}\right)=c_{j} \quad \text { with } \quad 1 \leq c_{j} \leq M_{j} \quad(j=1,2, \ldots)
$$

can be solved with a positive harmonic function $U$ on $\mathbb{R}_{+}^{n+1}$.

On the other hand, thin sequences in $\mathbb{R}_{+}^{n+1}$ can be defined in the spirit of Lemma 2.1. Namely, to each point $z_{j}=\left(x_{j}, y_{j}\right)$ of the sequence and to a fixed number $N \geq 1$ we associate the region

$$
S_{N, j}=\left\{(x, y) \in \mathbb{R}_{+}^{n+1}:\left|x-x_{j}\right|<\frac{1}{2} N y_{j}, 0<y<N y_{j}\right\}
$$

and we let $\mathcal{F}(N, j)$ denote the set of those indices $k \in \mathbb{N} \backslash\{j\}$ for which $z_{k} \in S_{N, j}$. This done, we say that $\left\{z_{j}\right\}$ is a thin sequence (in $\mathbb{R}_{+}^{n+1}$ ) if, for every $N \geq 1$,

$$
\lim _{j \rightarrow \infty} y_{j}^{-n} \sum_{k \in \mathcal{F}(N, j)} y_{k}^{n}=0
$$

Are thin sequences precisely the interpolating sequences with property $(*)$ ? We know that the answer is 'yes' when $n=1$, and our proof of the implication $(*) \Longrightarrow$ "thin" (i.e., implication (iv) $\Longrightarrow$ (i) in Theorem 1.5) seems to carry over to higher dimensions. The converse, if true, would call for a new approach.

\section{REFERENCES}

[1] L. Carleson and J. B. Garnett, Interpolating sequences and separation properties, J. Anal. Math. 28 (1975), 273-299.

[2] K. M. Dyakonov, Moment problems for bounded functions, Comm. Anal. Geom. 2 (1994), 533-562. MR1336894 (96d:41004)

[3] J. P. Earl, On the interpolation of bounded sequences by bounded functions, J. London Math. Soc. 2 (1970), 544-548. MR0284588 (44:1813)

[4] J. B. Garnett, Bounded Analytic Functions, Academic Press, New York, 1981. MR0628971 (83g:30037)

[5] P. Gorkin and R. Mortini, Asymptotic interpolating sequences in uniform algebras, J. London Math. Soc. 67 (2003), 481-498. MR.1956148 (2003k:46072)

[6] A. Hartmann, X. Massaneda, A. Nicolau and P. Thomas, Interpolation in the Nevanlinna and Smirnov classes and harmonic majorants, J. Funct. Anal. 217 (2004), 1-37. MR2097605 (2005h:30065)

[7] P. Jones, Estimates for the corona problem, J. Funct. Anal. 39 (1980), 162-181. MR0597809 (82i:30051)

[8] P. Koosis, Carleson's interpolation theorem deduced from a result of Pick, in: Complex analysis, operators, and related topics, 151-162, Oper. Theory Adv. Appl., 113, Birkhäuser, Basel, 2000. MR 1771759 (2002a:30061)

[9] A. Nicolau, Interpolating Blaschke products solving Pick-Nevanlinna problems, J. Anal. Math. 62 (1994), 199-224. MR.1269205 (94m:30071) 
[10] C. Sundberg and T. Wolff, Interpolating sequences for $Q A_{B}$, Trans. Amer. Math. Soc. 276 (1983), 551-581. MR0688962 (84e:30078)

[11] T. Wolff, Some theorems on vanishing mean oscillation, Thesis, Univ. of California, Berkeley, California, 1979.

iCREA and Departament de Matemàtica Aplicada i AnÀlisi, Universitat de Barcelona, Gran Via 585, E-08007 Barcelona, Spain

E-mail address: dyakonov@mat.ub.es

Departament de Matemàtiques, Universitat Autònoma de Barcelona, E-08193 BelLATERRA (BARCELONA), SpAin

E-mail address: artur@mat.uab.es 